\title{
Accurate determination of surface normal stress in viscous flow from a consistent boundary flux method
}

\author{
Shijie Zhong ${ }^{*, a}$, Michael Gurnis ${ }^{\text {a }}$, Gregory Hulbert ${ }^{\mathrm{b}}$ \\ ${ }^{a}$ Department of Geological Sciences, ${ }^{b}$ Department of Mechanical Engineering and Applied Mechanics, The University of Michigan, \\ Ann Arbor, MI 48109-1063, USA
}

(Received 8 October 1992; revision accepted 27 January 1993)

\begin{abstract}
Accurate determination of surface normal stresses from numerical modeling of mantle convection is crucial in determining surface topography, geoid and gravity anomalies. With the finite element method, we have developed a consistent boundary flux (CBF) method for computing the surface stress by solving the momentum equation directly. The method has a much higher accuracy for determining surface stresses than the standard pressure smoothing method, and for typical convection problems, the CBF is about one order of magnitude more accurate than pressure smoothing. The CBF can be easily applied to a variety of types of elements and to compute a range of physical quantities including heat flow on boundaries. CBF, moreover, is a post-processing operation and is computationally inexpensive.
\end{abstract}

\section{Introduction}

Topography, geoid and gravity are among the most important observations constraining thermal convection within the Earth's mantle. Numerical modeling of thermal convection reveals the fundamental relationship between the surface observables and internal dynamics. As the geoid and gravity both directly depend on surface deformation (dynamic topography) induced by thermal convection, accurate computation of dynamic topography is essential for determining all three observables. Dynamic topography can be determined from normal stresses acting on surfaces, assuming that the normal stresses are compensated through surface deformation on free surfaces.

With the Boussinesq approximation, the Earth's mantle can be regarded as an incompressible viscous fluid. Viscous flow can be solved using the finite element method (FEM) for complicated geometries and rheologies. To solve for

* Corresponding author. an incompressible viscous flow, the standard FEM uses a penalty function formulation with velocity as a primary variable (Hughes, 1987). Because of the simplicity of this formulation, it has been used widely in mantle convection studies (Schubert and Anderson, 1985; King et al., 1990). Also, it allows geodynamicists to study many important aspects in mantle convection, including the effects of faults (Zhong and Gurnis, 1992). The standard method to compute the surface stress in the FEM is pressure smoothing (Hughes, 1987, pp. 226-231). The pressure smoothing method computes the averaged stress for each element with a constitutive equation, and then projects this averaged stress onto adjacent nodes. For boundary nodes, a linear extrapolation is used to adjust the stress. Although the pressure smoothing method works very well for interior nodes (indeed, the pressure smoothing is a least-square approximation of stresses for interior nodes) (Hughes, 1987, pp. 297-303), its accuracy degenerates on boundaries (King, 1991). The loss of accuracy for boundary stresses becomes more serious when a heterogeneous rheology is present,

0031-9201/93/\$06.00 (C) 1993 - Elsevier Science Publishers B.V. All rights reserved 
such as a temperature-dependent viscosity which may be fundamentally important for the mantle.

Here we introduce a new method, consistent boundary flux calculation (CBF), originally proposed by Mizukami (1986) and Hughes (1987), to compute boundary stresses. With the CBF, boundary stresses are computed by solving the momentum equation within boundary row elements, and thus a higher accuracy is achieved compared with the pressure smoothing, where stresses are calculated with a constitutive equation. The CBF does not require any modification in solving the flow field and thus can be carried out during post processing. Without increasing computational complexity, we can determine the stress on boundaries where velocities are prescribed as boundary conditions with much higher accuracy than pressure smoothing. The finite element code, ConMan (King et al., 1990), which employs bilinear quadrilateral elements, is used to solve the velocity field. We will first review how the CBF works, and then show several examples. We will compare dynamic topography and geoid from the $\mathrm{CBF}$ and the pressure smoothing with both analytic solutions and a recent convection benchmark (Blankenbach et al., 1989). Although bilinear elements are used, the CBF can be applied to various types of elements. The CBF can also be applied to computing surface heat flow (Nusselt number) of convection with a higher accuracy than pressure smoothing (e.g. Ho-Liu et al., 1987).

\section{Method}

Surface stresses are determined by the momentum and continuity equations, which, for an incompressible viscous fluid with no inertial forces, are

$\sigma_{i j, j}+f_{i}=0$

and

$u_{i, i}=0$

where $f_{i}$ is the body force, $\sigma_{i j}$ is the stress tensor, and $u_{i}$ is the flow velocity. Throughout the paper, repeated indices denote summation.

The boundary conditions are in general a combination of prescribed stress and velocity and can be expressed as

$u_{i}=g_{i}$ on $\Gamma_{g_{i}}$

and

$\sigma_{i j} n_{j}=h_{i}$ on $\Gamma_{h_{i}}$

where $\Gamma_{g_{i}}$ is the boundary over which the flow velocity is prescribed and $\Gamma_{h_{i}}$ is the boundary on which traction is given. We assume a linear constitutive equation

$\sigma_{i j}=-p \delta_{i j}+\mu\left(u_{i, j}+u_{j, i}\right)$

where $\delta_{i j}$ is the Kronecker delta, $p$ is the pressure and $\mu$ is the dynamic viscosity.

Equations (1)-(4) can be solved for an irregular geometry and a spatially variable rheology with a penalty function formulation (Hughes, 1987). The Galerkin weak formulation can be stated as: find $u_{i}=v_{i}+g_{i}$ and $v_{i} \in U^{h}$, where $U^{h}$ is a set of functions in which each function, $w_{i}$, is equal to zero on $\Gamma_{g_{i}}$, such that for all $w_{i} \in \boldsymbol{U}^{h}$

$$
\begin{aligned}
\int_{\Omega} w_{i, j} c_{i j k l} v_{k, l} \mathrm{~d} \Omega= & \int_{\Omega} w_{i} f_{i} \mathrm{~d} \Omega+\sum_{i=1}^{n_{\mathrm{sd}}} \int_{\Gamma_{h_{i}}} w_{i} h_{i} \mathrm{~d} \Gamma \\
& -\int_{\Omega} w_{i, j} c_{i j k l} g_{k, l} \mathrm{~d} \Omega
\end{aligned}
$$

where $n_{\text {sd }}$ is the number of space dimensions and $c_{i j k l}$ are the coefficients of the constitutive equation. The pressure smoothing method, using the velocity solved from (6) and the constitutive equation, Eq. (5), computes the element average $\sigma_{i j}$, and then projects the average $\sigma_{i j}$ onto boundary nodes.

With the CBF method, a function, $w_{i} \in U^{h}$, is not assumed to be zero on $\Gamma_{g_{i}}$; hence the equivalent form of (6) is

$$
\begin{aligned}
\int_{\Omega} w_{i, j} c_{i j k l} u_{k, l} \mathrm{~d} \Omega= & \int_{\Omega} w_{i} f_{i} \mathrm{~d} \Omega+\sum_{i=1}^{n_{\mathrm{sd}}} \int_{\Gamma_{h_{i}}} w_{i} h_{i} \mathrm{~d} \Gamma \\
& +\sum_{i=1}^{n_{\mathrm{sd}}} \int_{\Gamma_{g_{i}}} w_{i} s_{i} \mathrm{~d} \Gamma
\end{aligned}
$$




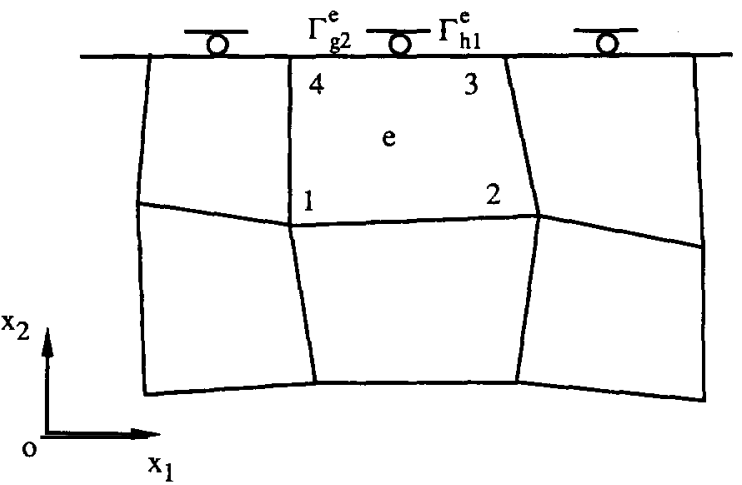

Fig. 1. In a coordinate system, $X_{1} O X_{2}$, a free slip boundary condition on the surface is $u_{2}=\sigma_{12}=0$, and for a quadrilateral element $e$, this leads to $\Gamma_{g_{2}}^{e} \equiv \Gamma_{h_{1}}^{e}$. The numbers are the local node numbers.

where $s_{i}$ are the unknown tractions on $\Gamma_{g_{i}}$ and can be expressed as

$s_{i}\left(x_{i}\right)=\sum_{a \in \Gamma_{g_{i}}} N_{a}\left(x_{i}\right) \tilde{s}_{a}$

where $\tilde{s}_{a}$ is the unknown traction at boundary node $a$. As $N_{a}$ in Eq. (8) is zero for a node that is not on $\Gamma_{g_{i}}$, it is easy to show that the equations which govern the flow field remain unchanged from (6). Using the flow field solved from (6) and substituting (8) into (7) yields a set of equations computing the surface traction, $\tilde{s}_{a}$, on $\Gamma_{g_{i}}$. This procedure can be carried out at an element level. Only elements connected to boundary $\Gamma_{g_{i}}$ need to be computed. This approach will be illustrated with the following example. Let us suppose that for an element $e$, the boundary side is prescribed with a horizontal traction and a vertical velocity (Fig. 1), i.e. $\Gamma_{g_{2}}^{e} \equiv \Gamma_{h_{1}}^{e}$ A matrix form of Eq. (7) for the element $e$ can be written as

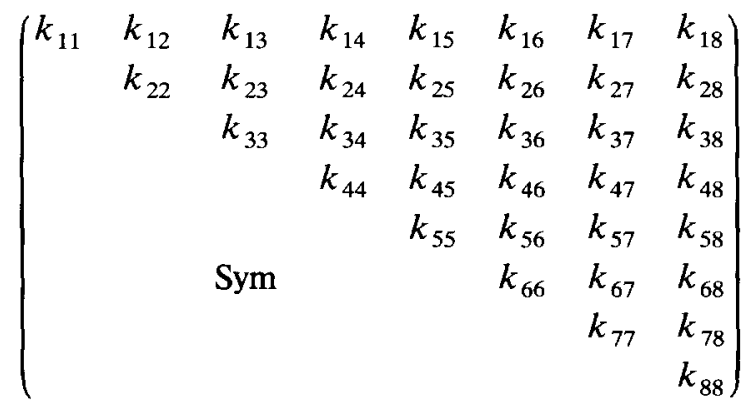

$$
\times\left(\begin{array}{l}
u_{1} \\
u_{2} \\
u_{3} \\
u_{4} \\
u_{5} \\
u_{6} \\
u_{7} \\
u_{8}
\end{array}\right)=\int_{\Omega_{e}}\left(\begin{array}{l}
N_{1} f_{1} \\
N_{1} f_{2} \\
N_{2} f_{1} \\
N_{2} f_{2} \\
N_{3} f_{1} \\
N_{3} f_{2} \\
N_{4} f_{1} \\
N_{4} f_{2}
\end{array}\right) \mathrm{d} \Omega+\int_{\Gamma_{g_{2}}^{e}}\left(\begin{array}{c}
0 \\
0 \\
0 \\
0 \\
N_{3} h_{1} \\
N_{3} s_{2} \\
N_{4} h_{1} \\
N_{4} s_{2}
\end{array}\right) \mathrm{d} \Gamma
$$

where the $k_{i j}$ are entries of an element stiffness matrix, the $N_{a}$ are shape functions, $h_{1}$ is the prescribed horizontal traction, and $s_{2}$ is the unknown normal stress on $\Gamma_{g_{2}}^{e}$. Whereas the first five and the seventh sub-equations of (9) are simply parts of the normal equations of motion (i.e. Eq. (6)), the sixth and the eighth sub-equations form equations which solve for the normal stress on nodes 3 and 4 (Fig. 1). After substituting (8) into (9), the sixth and the eighth sub-equations of (9) can be expressed as

$$
\begin{aligned}
& \frac{2}{l_{e}}\left(\begin{array}{cc}
\int_{-1}^{1} \tilde{N}_{3} \tilde{N}_{3} \mathrm{~d} \xi & \int_{-1}^{1} \tilde{N}_{3} \tilde{N}_{4} \mathrm{~d} \xi \\
\int_{-1}^{1} \tilde{N}_{3} \tilde{N}_{4} \mathrm{~d} \xi & \int_{-1}^{1} \tilde{N}_{4} \tilde{N}_{4} \mathrm{~d} \xi
\end{array}\right)\left(\begin{array}{c}
\tilde{s}_{3} \\
\tilde{s}_{4}
\end{array}\right) \\
& =\left(\begin{array}{c}
\sum_{j=1}^{8} k_{6 j} u_{j}-\int_{\Omega_{e}} N_{3} f_{2} \mathrm{~d} \Omega \\
\sum_{j=1}^{8} k_{8 j} u_{j}-\sum_{\Omega_{e}} N_{4} f_{2} \mathrm{~d} \Omega
\end{array}\right)
\end{aligned}
$$

where an isoparametric element notation (e.g. Hughes, 1987) is used for the left-hand side, $l_{e}$ is the length of $\Gamma_{g_{2}}^{e}$, and $\tilde{N}_{3}$ and $\tilde{N}_{4}$ are the degenerated one-dimensional shape functions, which can be written as

$\tilde{N}_{i}=\frac{1}{2}\left(1+\xi_{i} \xi\right)$

where $\xi_{i}=-1$ for $i=3$ and $\xi_{i}=1$ for $i=4$. The degeneration of the shape functions results from the fact that the second integration on the righthand side of (9) is along $\Gamma_{g_{2}}^{e}$.

Equation (10) can be assembled for all the boundary elements in the usual way, and thus a set of equations with the unknown boundary normal stresses is formed. The $2 \times 2$ matrix in (10), 
given the shape functions in (11), can be analytically computed and the diagonal entries and the off-diagonal entries are $1 / 3$ and $1 / 6$, respectively. After being assembled, the global matrix equation is a tri-diagonal matrix equation and can be easily solved with a conjugate gradient method. With a trapezoidal integration rule, the $2 \times 2$ matrix can even be diagonalized (Hughes, 1987), and the resulting equations can be solved very efficiently without losing much accuracy. We have used the former approach in this paper.

The CBF occurs during a post-processing phase, and does not have any effect on the primary equations of the flow. The CBF clearly has several advantages over the pressure smoothing. For instance, the CBF computes the boundary stress directly on the boundaries, therefore this method works for various irregular elements; the pressure smoothing method prefers rectangle elements, because extrapolation of the elemental stress onto the boundaries is used. Also, by solving the momentum equation, the CBF automatically includes the contribution of the body force within the boundary row elements to the boundary stress. The pressure smoothing method cannot accurately account for the contribution of the body force (King (1991) proposed a different approach to include the contribution of the body force). For thermal convection problems, because of the existence of thermal boundary layers, the body force may be significant in determining the surface stress. It should be pointed out, however, that the CBF can only be used to compute boundary stresses. For some problems, such as thermal convection with a stress-dependent rheology, in which computing the interior stresses is necessary, the pressure smoothing method is still needed.

\section{Results and discussion}

The advantage of the CBF over the pressure smoothing method will be illustrated through three test cases. The first case is an analytic solution and the other two are the standard cases used in mantle convection benchmarks (Blankenbach et al., 1989). For all three cases, the improvement in computing boundary stress is significant, especially for the case with variable viscosity.

In Case 1 , an isoviscous flow within a $1 \times 1$ box with free slip boundary conditions on all boundaries is driven by a buoyant force

$f_{1}=0$ and $f_{2}=\rho \alpha g T\left(x_{1}, x_{2}\right)$

where $\rho$ and $\alpha$ are the density and the coefficient of thermal expansion, respectively, $g$ is the acceleration of gravity, and $T\left(x_{1}, x_{2}\right)$ is the temperature field, given by

$$
\begin{aligned}
T\left(x_{1}, x_{2}\right) & =\cos \left(k x_{1}\right) \cdot \delta\left(x_{2}-x_{2}^{\prime}\right), \\
& \text { for } 0 \leq x_{1}, x_{2} \leq 1
\end{aligned}
$$

where $k=2 \pi / \lambda$ and $\lambda$ is a wavelength, $\delta\left(x_{2}-\right.$ $\left.x_{2}^{\prime}\right)$ is the Dirac delta function, and $x_{2}^{\prime}$ represents the location of the buoyancy. Using a Green's function method (Parson and Daly, 1983), an analytic solution of surface stress $\sigma_{22}$, after being normalized by $\rho \alpha g$, can be obtained:

$$
\begin{aligned}
& \sigma_{22}\left(x_{1}, 1\right)= \frac{\cos \left(k x_{1}\right)}{\sinh ^{2}(k)}\left\{k\left(1-x_{2}^{\prime}\right) \sinh (k)\right. \\
& \times \cosh \left(k x_{2}^{\prime}\right)-k \sinh \left[k\left(1-x_{2}^{\prime}\right)\right] \\
&\left.+\sinh (k) \sinh \left(k x_{2}^{\prime}\right)\right\}, \\
& \text { for } 0 \leq x_{1} \leq 1
\end{aligned}
$$

TABLE 1

Surface stress $\sigma_{22}$ at the top-left corner point $\left(x_{1}=0\right)$ for Case 1

\begin{tabular}{llll}
\hline Method & $x_{2}^{\prime}=63 / 64$ & $x_{2}^{\prime}=62 / 64$ & $x_{2}^{\prime}=60 / 64$ \\
\hline Analytic solution & 0.995476 & 0.983053 & 0.912506 \\
Pressure smoothing & $1.15974(16.5 \%)$ & $1.06498(8.3 \%)$ & $0.911109(0.15 \%)$ \\
CBF & $0.994236(0.13 \%)$ & $0.982116(0.10 \%)$ & $0.912157(0.04 \%)$ \\
\hline
\end{tabular}


TABLE 2

Physical and geometry parameters for Cases 2 and 3

\begin{tabular}{ll}
\hline $\begin{array}{l}\text { Height, } h \text {, and length, } l, \\
\quad \text { of the box }\end{array}$ & $10^{6} \mathrm{~m}$ \\
Temperature contrast, $\Delta T$ & $1000 \mathrm{~K}$ \\
Density, $\rho$ & $4 \times 10^{3} \mathrm{~kg} \mathrm{~m}^{-3}$ \\
Thermal diffusivity & $10^{-6} \mathrm{~m}^{2} \mathrm{~s}^{-1}$ \\
Acceleration of gravity, $g$ & $10 \mathrm{~m} \mathrm{~s}^{-2}$ \\
Thermal expansion, $\alpha$ & $2.5 \times 10^{-5} \mathrm{~K}^{-1}$ \\
(Surface) kinematic viscosity, $\nu_{0}$ & $2.5 \times 10^{19} \mathrm{~m}^{2} \mathrm{~s}^{-1}$ \\
Gravitational constant & $6.673 \times 10^{-11} \mathrm{~N} \mathrm{~m}^{2} \mathrm{~kg}^{-2}$
\end{tabular}

For $\lambda=1$, the surface stresses $\sigma_{22}$ for $x_{2}^{\prime}=$ $63 / 64,62 / 64$, and $60 / 64$ are computed. The $\sigma_{22}$ values at the top-left corner point $\left(x_{1}=0\right)$ approach one, as the buoyancy is approaching the surface (Table 1).

With the FEM, the flow with the same buoyancy is solved for a $64 \times 64$ uniform mesh, and $\sigma_{22}$ on the surface is computed with both the pressure smoothing method and the CBF (Table 1). Although the pressure smoothing is fairly accurate when the buoyancy is four meshes away from the surface, the accuracy from the pressure smoothing suffers when the buoyancy is one or two meshes away from the surface; a similar result was also reported by King (1991). The CBF is clearly superior to the pressure smoothing (Table 1). For example, when the buoyancy is only one mesh away from the surface $\left(x_{2}^{\prime}=63 / 64\right)$, the relative error (Table 1, in parentheses) of the pressure smoothing is $16.5 \%$, but the CBF reduces the error to $0.13 \%$.
Cases 2 and 3 are for full thermal convection problems involving the solution of the energy equation. We use a Streamline Upwind PetrovGalerkin method (Brooks, 1981) to solve the energy equation. Cases 2 and 3 are identical to Cases 1(a) and 2(a) in the Blankenbach et al. (1989) benchmark paper and are briefly defined below.

The flows are confined to a non-dimensional $1 \times 1$ box with free slip and isothermal boundary conditions on the bottom and top boundaries and with reflecting boundary conditions on the vertical boundaries. The body force also has the form of (12). For Case 2, a constant viscosity with a Rayleigh number of $10^{4}$ is used. For Case 3, a temperature-dependent viscosity,

$\nu=\nu_{0} \exp \left(-\frac{b T}{\Delta T}\right)$

is used. A Rayleigh number defined by surface viscosity, $\nu_{0}$, is also $10^{4}$. In (15), $b=\ln (1000)$, which limits the maximum variation of viscosity to $10^{3}$, and $\Delta T$ is the temperature difference between bottom and top boundaries. An initial condition is chosen such that a single convection cell forms in the steady state.

Topography can be calculated from the surface normal stress by assuming that the surface will deform with the normal stress such that no net traction acts on the surface after deforming. Following Blankenbach et al. (1989), the topography and geoid are computed by setting the mean value of each to zero. Both calculations are done

TABLE 3

Topography and geoid for Case 2 (numbers in parentheses are relative errors, given as percentages)

\begin{tabular}{llllll}
\hline Mesh & & $\xi_{1}$ & $\xi_{2}$ & $\Psi_{1}$ & $\Psi_{2}$ \\
\hline $24 \times 24$ & CBF & $2256.7(0.12)$ & $-2906.6(0.11)$ & $54.92(0.18)$ & $-62.72(0.15)$ \\
& PS & $2283.1(1.30)$ & $-2930.2(0.93)$ & $56.64(3.30)$ & $-64.46(2.90)$ \\
$32 \times 32$ & CBF & $2255.5(0.07)$ & $-2907.5(0.15)$ & $54.89(0.08)$ & $-62.71(0.15)$ \\
& PS & $2272.0(0.80)$ & $-2922.1(0.65)$ & $55.94(2.00)$ & $-63.77(1.80)$ \\
$48 \times 48$ & CBF & $2254.7(0.03)$ & $-2905.2(0.07)$ & $54.85(0.06)$ & $-62.67(0.08)$ \\
& PS & $2262.6(0.38)$ & $-2912.1(0.31)$ & $55.35(0.97)$ & $-63.17(0.87)$ \\
$64 \times 64$ & CBF & $2254.4(0.02)$ & $-2904.3(0.04)$ & $54.84(0.03)$ & $-62.65(0.04)$ \\
& PS & $2259.0(0.22)$ & $-2908.3(0.18)$ & $55.12(0.55)$ & $-62.93(0.49)$ \\
Christensen & & 2254.0 & -2903.2 & 54.82 & -62.62 \\
\hline
\end{tabular}


dimensionally with values shown in Table 2 . In computing the topography, no overlying medium is assumed, and the density of the underlying medium is twice that of the flow. The geoid is computed with a spectral method.

For Case 2, surface topographies at $x_{1}=0, \xi_{1}$, and at $x_{1}=1, \xi_{2}$, from pressure smoothing and CBF methods with different meshes, and Christensen's extrapolated results given by Blankenbach et al. (1989) are shown in Table 3. The relative errors of the pressure smoothing and the CBF (Table 3, in parentheses) are also computed by taking Christensen's extrapolated results as 'exact' solutions. The pressure smoothing method (referred to as PS in Table 3 ) achieves a reasonably high accuracy, and typical relative errors are about $1 \%$. However, the CBF has a much higher accuracy and reduces the relative errors by about one order of magnitude (Table 3). The geoid is the difference between two terms: one measures contributions from internal density structure and another accounts for the topographic contribution. As these two terms usually have much larger magnitudes than the geoid itself, the geoid is very sensitive to each of them; accurate topography is essential for an accurate determination of the geoid. The geoid at $x_{1}=0, \Psi_{1}$, and at $x_{1}=1, \Psi_{2}$ (Table 3), clearly shows that the CBF yields a much more accurate geoid than pressure smoothing. For example, for a $32 \times 32$ mesh, whereas the relative error of $\Psi_{1}$ is $2.0 \%$ for the pressure smoothing method, it is reduced to $0.08 \%$ for the CBF.

For Case 3 with a temperature-dependent viscosity, we observe (Table 4) that the pressure smoothing method significantly underestimates topography, especially over thermal upwellings. For example, for a $48 \times 48$ mesh, the relative error of topography over the upwelling $\left(x_{1}=0\right)$ is $23 \%$, and this error is notably larger than that over the downwelling $\left(x_{1}=1\right)$, which is $4.70 \%$. Also, accuracy of the CBF is about one order of magnitude higher than that of the pressure smoothing. Not surprisingly, geoids from the CBF have much smaller errors than those from the pressure smoothing method. The differences in surface topography and the geoid between these two methods can easily be seen in Fig. 2, which shows the topography and geoid profiles for a $48 \times 48$ mesh.

The pressure smoothing method significantly underestimates topography for Case 3 with a temperature-dependent viscosity. As is well known, temperature-dependent viscosity can thin the upper thermal boundary layer, especially near upwellings. This results in a sharp change in viscosity and body forces near the boundaries. Any difference scheme, which is necessary for computing the stress with a constitutive equation in the pressure smoothing method, will be inaccurate near these boundaries. The accuracy becomes worse near the upwelling, as a result of the thinner boundary layers, and this explains why the errors over the upwellings are much larger than those over the downwellings (Table 4). However, the CBF overcomes this difficulty by solving the boundary stress directly from the momentum equation. A higher Rayleigh number would result in an even thinner thermal boundary layer, and thus the CBF has a great advantage. With a $64 \times 64$ mesh substantially refined near the top and bottom boundaries, we have computed to-

TABLE 4

Topography and geoid for Case 3 (numbers in parentheses are relative errors, given as percentages)

\begin{tabular}{llclll}
\hline Mesh & & $\xi_{1}$ & $\xi_{2}$ & $\Psi_{1}$ & $\Psi_{2}$ \\
\hline $32 \times 32$ & CBF & $1044.4(3.30)$ & $-4029.2(1.70)$ & $18.50(6.70)$ & $-54.98(0.69)$ \\
& PS & $721.36(28.6)$ & $-3752.1(8.40)$ & $6.064(65.0)$ & $-47.01(13.9)$ \\
$48 \times 48$ & CBF & $1023.6(1.26)$ & $-4062.4(0.87)$ & $17.78(2.50)$ & $-54.71(0.20)$ \\
& PS & $778.16(23.0)$ & $-3906.5(4.70)$ & $8.497(51.0)$ & $-49.28(9.70)$ \\
$64 \times 64$ & CBF & $1019.3(0.80)$ & $-4084.4(0.33)$ & $17.67(1.90)$ & $-54.61(0.02)$ \\
& PS & $833.19(17.6)$ & $-3982.5(2.82)$ & $10.68(38.4)$ & $-50.83(6.90)$ \\
Christensen & & 1010.9 & -4098.1 & 17.34 & -54.60 \\
\hline
\end{tabular}


a)

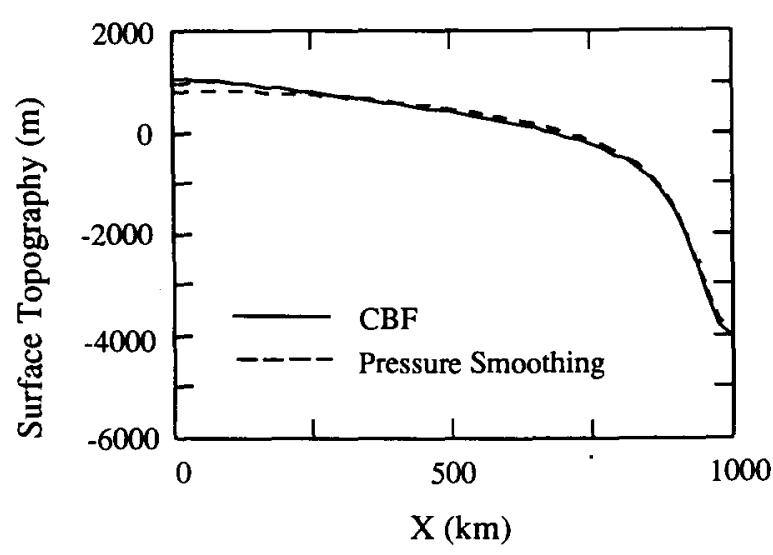

b)

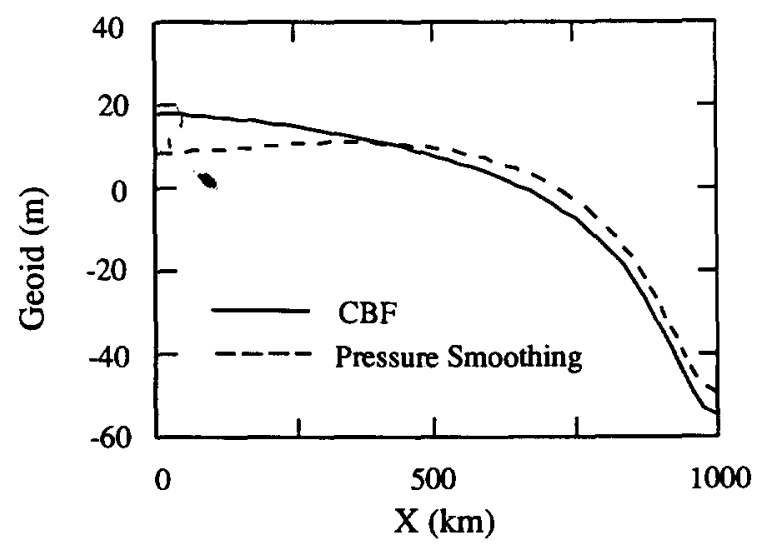

Fig. 2. Comparison of surface topography and geoid between the pressure smoothing method (dashed lines) and the CBF (solid lines) for Case 3 with a $48 \times 48$ mesh.

pography and geoid for a case which is identical to Case 3 except now $\mathrm{Ra}=10^{5}$. The $\xi_{1}$ and $\xi_{2}$ are $744.69 \mathrm{~m}$ and $-2803.6 \mathrm{~m}$ from the $\mathrm{CBF}$, and $525.64 \mathrm{~m}$ and $-2721.4 \mathrm{~m}$ from the pressure smoothing, respectively. The pressure smoothing method underestimates $\xi_{1}$ and $\xi_{2}$ by about $30 \%$ and $3.0 \%$, respectively.

It should be pointed out that there are still some differences between Christensen's results (Blankenbach et al., 1989) and those from the CBF. Several factors may be responsible for these differences: (1) although both of us have used finite element methods, our formulations are different - Christensen (1984) used a streamfunction-vorticity formulation, whereas we have used a penalty function formulation; (2) Christensen used refined and bicubic elements, whereas we used uniform and bilinear elements; (3) in searching for a steady state, Christensen directly solved static equations, whereas we solve the time-dependent equations.

In summary, the CBF acquires about one order of magnitude higher accuracy than the pressure smoothing method in computing boundary stress without increasing computational cost, and thus yields more accurate topography and geoid. The CBF can easily be applied to a range of element types, and to compute various physical quantities such as heat flow on boundaries.

\section{Acknowledgments}

We thank Dr. Scott King for his reviews and his suggestion that we should study Case 1 . Computations were done on the Cray YMP at the Pittsburgh Supercomputer Center. This work was funded by the David and Lucile Packard Foundation and NSF grants EAR-8957164 and EAR8904660 .

\section{References}

Blankenbach, B., Busse, F., Christensen, U., Cserepes, L., Gunkel, D., Hansen, U., Harder, H., Jarvis, G., Koch, M., Marquart, G., Moore, D., Olsen, P., Schmeling, H. and Schnaubelt, T., 1989. A benchmark comparison for mantle convection codes. Geophys. J. Int., 98: 23-38.

Brooks, A.N., 1981. A Petrov-Galerkin finite element formulation for convection dominated flows. PhD thesis, California Institute of Technology, Pasadena, CA.

Christensen, U., 1984. Convection with pressure- and temperature-dependent non-Newtonian rheology. Geophys. J. R. Astron. Soc., 77: 343-384.

Ho-Liu, P., Hager, B.H. and Raefsky, A., 1987. An improved method of Nusselt number calculation. Geophys. J. R. Astron. Soc., 88: 205-215.

Hughes, T.J.R., 1987. The Finite Element Method. PrenticeHall, Englewood Cliffs, NJ, pp. 631.

King, S.D., 1991. The interaction of subducting slabs and the 670 kilometer discontinuity. Ph.D. Thesis, California Institute of Technology, Pasadena, CA, pp. 142-147. 
King, S.D., Raefsky, A. and Hager, B.H., 1990. ConMan: vectorizing a finite element code for incompressible twodimensional convection in the Earth's mantle. Phys. Earth Planet. Inter., 59: 195-207.

Mizukami, A., 1986. A mixed finite element method for boundary flux computation. Comput. Meth. Appl. Mech. Eng., 57: 239-243.

Parson, B. and Daly, S., 1983. The relationship between surface topography, gravity anomalies, and temperature structure of convection. J. Geophys. Res., 88: 1129-1144.
Schubert, G. and Anderson, C.A., 1985. Finite element calculations of very high Rayleigh number thermal convection. Geophys. J. R. Astron. Soc., 80: 575-601.

Zhong, S. and Gurnis, M., 1992. Viscous flow model of a subduction zone with a faulted lithosphere: long and short wavelength topography, gravity and geoid. Geophys. Res. Lett., 19: 1891-1894. 